\title{
Violencia y maltrato en la familia romana y sus consecuencias: la adolescencia perdida de Tiberio Claudio
}

\author{
Violence and maltreatment on the roman family and their \\ consequences: the Tiberius Claudius' case
}

\author{
MILAGROS MORO IPOLA* \\ Universidad Nacional de Educación a Distancia - UNED
}

\begin{abstract}
RESUMEN
El maltrato psicológico es todo aquel que se ejerce contra el normal desarrollo de la personalidad de un niño. Aunque actual, es una situación que niños y adolescentes han venido sufriendo a lo largo de los siglos y cuyas consecuencias se han dejado ver, ya no sólo durante su adolescencia, sino también en la edad adulta. Uno de los casos más significativos de maltrato hacia un menor es el que sufrió el emperador Tiberio
\end{abstract}

Claudio.

\section{PALABRAS CLAVE}

Tiberio Claudio, familia, maltrato, abuso, secuelas, enfermedad.

\begin{abstract}
The psychological maltreatment is whatever is exercised against the normal development of the personality of a child. Even though it is a current situation, children and teenagers have been suffering it over the centuries and its consequences can be seen not only during the adolescence but in their manhood. One of the most significant cases of maltreatment against a kid is that one Emperor Tiberius Claudius suffered.
\end{abstract}

\section{KEYWORDS}

Tiberius Claudius, family, maltreatment, abuse, consequences, illness.

\section{INTRODUCCIÓN}

En la conducta adolescente influyen dos tipos de factores: biológicos y socioculturales.

Los últimos estudios realizados sobre el funcionamiento del cerebro en adolescentes ${ }^{1}$ revelan las diferencias neurológicas que existen entre los cerebros de

* E-mail: nani_poppins@ hotmail.com. Dirección postal: C/Norte, 30, 8ª 46008 Valencia (España).

1 WALLIS, C.: 2004. 
los adolescentes y los de los adultos. El que el proceso de maduración del cerebro se prolongue hasta los veinticinco años y que comience en la parte posterior, en el lóbulo Occipital, siendo la corteza pre-frontal la última en hacerlo, tiene como primera consecuencia que adultos y adolescentes ante el mismo estímulo utilicen para procesar e interpretar la información partes diferentes de sus cerebros. Mientras los adultos emplean más el lóbulo frontal, los adolescentes tienen una mayor actividad en la amígdala que es donde residen sensaciones como el miedo o la cólera y ese mayor uso explica parte de su comportamiento como los brotes de ira o las reacciones impulsivas. Esta especial configuración de sus cerebros y la mayor actividad de otros órganos, como el nucleus accumbens ${ }^{2}$, por ejemplo, serían los responsables de la necesidad que tienen los adolescentes por la gratificación inmediata o su falta de empatía ante los sentimientos de los demás.

Pero si los condicionamientos neurológicos son, sin duda, importantes los que realmente son determinantes en el comportamiento de los adolescentes son los culturales: la familia, el grupo de iguales, la sociedad. $Y$ de entre todos ellos, el fundamental y más decisivo es la familia. Hoy, al igual que hace dos mil años, el lugar dónde nacemos, status social, la educación recibida y, sobre todo, el ejemplo paterno condiciona nuestra conducta ya no sólo durante la adolescencia sino también durante la vida adulta.

Si en circunstancias que podríamos llamar «normales» la influencia familiar es evidente, qué decir en casos de divorcio, adopción o violencia. Los malos tratos, tanto físicos como psíquicos, el abuso sexual, el abandono afectivo, producen en los hijos tales heridas que difícilmente las pueden superar al llegar a la madurez condicionando irremediablemente su conducta para el resto de su vida.

Esa situación la vivieron muchos jóvenes romanos, incluidos algunos miembros de la propia familia imperial. Y ahí, en el Palatino, es dónde encontramos el caso que vamos a ver.

Debido a sus problemas físicos, Tiberio Claudio fue humillado, maltratado, menospreciado, agredido, insultado, ignorado y amenazado por su propia familia desde que nació. En este estudio intentaremos comprender por qué fue tratado de esa manera y cómo eso afectó a su conducta como adulto.

\section{EL CASO DE TIBERIO CLAUDIO}

Tiberio Claudio Druso nació en Lugdunum (actual Lyon) en las calendas de agosto del año 10 a.C. durante el consulado de Julo Antonio y Fabio Africano ${ }^{3}$. Era hijo de Nerón Claudio Druso y de Antonia la menor y, por consiguiente, nieto de Livia, y de Marco Antonio y Octavia. Cuando nació, sus padres ya habían tenido varios hijos aunque únicamente habían sobrevivido dos: Julio César Germánico nacido en el año 15 a.C. y Claudia Livia Julia, Livila, en el 13 a.C.

2 El nucleus accumbens es un grupo de neuronas del encéfalo que tiene un papel importante en la recompensa, la risa, la adicción y el miedo. SCHWIENBACHER, I; FENDT, M.; RICHARDSON, R.; SCHNITZLER, H.:2004.

${ }^{3}$ Suet., $\mathrm{Cl}$. II. 
Probablemente el de Claudio fue un parto prematuro. Su propia madre en más de una ocasión lo definió como «un engendro humano que la naturaleza había dejado de terminar» ${ }^{4}$. En un alumbramiento de estas características, la probabilidad de que haya un sufrimiento fetal aumenta considerablemente y éste debió de ser uno de esos. Teniendo en cuenta los síntomas presentados por Claudio descritos por Suetonio, todo apunta a que en el momento del nacimiento, el bebé sufrió una isquemia, un corte del riego sanguíneo en el cerebro con la consiguiente falta de oxígeno que se tradujo en una necrosis del tejido neuronal ${ }^{5}$ produciéndose lo que hoy en día se conoce como Síndrome de Little, un tipo de parálisis cerebral infantil irreversible pero no progresivo ${ }^{6}$.

El cerebro del pequeño Claudio quedó probablemente dañado en su parte central que es donde se encuentra el sistema motor y el oído. En la figura que reproducimos a continuación, se corresponde a la zona señalada en un tono más oscuro.

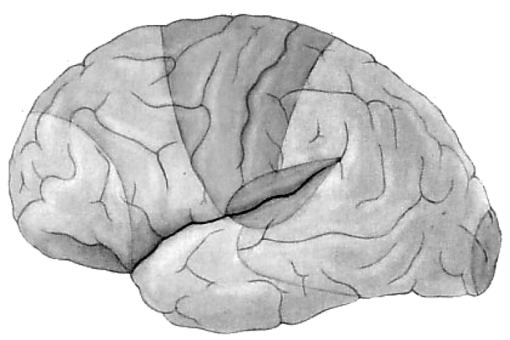

Fig. 1. Vista del cerebro. Sobotta, Atlas de anatomía Humana. Editorial Médica Panamericana, S.A., 2001.

4 Ídem, p. 2.

5 En 1947, el doctor Peter Dal Bianco, del departamento de neurología del hospital universitario de Innsbruck, ya establecía que los síntomas revelados por Claudio respondían a un síndrome neurológico. Un año más tarde, Ernestine Leon, profesor de la universidad de Texas, veía el origen de los problemas de Claudio en una lesión cerebral que se habría producido durante el parto o durante los primeros meses de vida y que afectaría a su aparato motriz aunque no a su inteligencia. Fue el profesor Grmek quién definitivamente confirmó que los síntomas de Claudio respondían a una encefalopatía infantil, muy probablemente, un síndrome de Little. La enfermedad podría estar documentada en Grecia a través de un pasaje de la Ética a Nicómaco dónde aparece un personaje que se desplaza con dificultad y que presenta las características piernas «en forma de tijeras». MARTIN, R.F.:1989.

${ }^{6}$ La Parálisis Cerebral Infantil (PCI) es un trastorno neuromotor identificado por primera vez por el doctor William Little a mitad del siglo XIX. Esta afección neurológica aparece durante los primeros meses de vida en bebés nacidos tras un parto difícil. En este tipo de partos se puede producir lo que en términos médicos se denomina una isquemia, es decir, una disminución del riego sanguíneo que provoca lesiones cerebrales más o menos intensas que determinan la amplitud de la enfermedad motriz cerebral. El resultado es una paraplejía espática y congénita que se traduce en problemas tales como: mala postura, movimientos involuntarios en los cuatro miembros aunque más pronunciados en los inferiores, rigidez muscular, espasmos, dificultad para hablar, caminar y/u oír, con frecuencia tartamudeo aunque la inteligencia, en el $80 \%$ de los casos, es normal o, incluso, superior. La parálisis cerebral es una lesión permanente aunque no progresiva lo que significa que no se agravará a medida que el niño vaya creciendo. Es necesario aclarar que la parálisis cerebral se manifiesta de diversas formas y que, mientras en algunas personas es apenas apreciable, otras pueden estar muy afectadas. Los bebés con más riesgo de padecer parálisis cerebral son aquellos prematuros que nacen sin sobrepasar las 37 semanas de gestación y con menos de 2.500 gr. de peso. http://acceso.uv.es/pci/PCI.html: 2007. Sobre la patología de Little: GREZE, J.: 1971; DENUELLE, P.:1974; http://salud.discapnet.es/: 2006. 
Claudio era un niño débil que perdía el equilibrio con facilidad, babeaba, moqueaba, sufría apraxia ${ }^{7}$, disquinesia ${ }^{8}$, distractibilidad, problemas de audición y dificultades en el habla y el lenguaje. Era incapaz de controlar los tics que sacudían su cabeza, tartamudeaba y, como no es extraño en los pacientes de $\mathrm{PCl}$, era epiléptico.

(...) al caminar, las rodillas le flaqueaban, pues las tenía débiles, y cuando desarrollaba cualquier actividad de recreo o en serio, muchos defectos le afeaban: una risa inconveniente, una cólera aún más indecorosa, que llenaba de espuma su boca dilatada y le humedecía las narices, un hablar entrecortado, y un tembleo de cabeza que, aun siendo constante, se acentuaba mucho más al menor gesto. (Suet., Cl. XXX).

Sin embargo, y a pesar de la gravedad de la lesión cerebral, su inteligencia no se vio afectada al quedarse fuera de la zona dañada.

Los primeros signos de abandono y desprecio los recibió de su propia madre que no solamente lo calificaba de engendro sino que, cuando quería expresar el grado de idiotez de alguna persona utilizaba a su hijo como comparación: Es más tonto que mi hijo Claudio ${ }^{9}$ (Suet., Cl. III).

Como le sucedía a gran parte de los niños romanos, Claudio perdió a su padre cuando apenas contaba un año de edad ${ }^{10}$. De forma que no contó con el ejemplo y la influencia que podía haber encontrado en Druso. Antonia, por su parte, no fue de ese tipo de madres romanas, como Aurelia o Atia, que habiendo enviudado, se hiciera cargo de la educación de los hijos. Antonia abandonó a su hijo en todos los aspectos, no solamente el afectivo, permitiendo que un niño de sus características tuviese como preceptor a un individuo que había sido anteriormente intendente de las caballerizas y que, como Claudio recordaría años más tarde, le trataba con la mayor crueldad posible sin la menor justificación (Suet., Cl. II 2).

Tampoco recibió de su abuela paterna ${ }^{11}$, Livia, afecto o atención. Todo lo contrario. La mujer de Octavio evitaba en lo posible cualquier contacto con él, comunicándose por medio de cartas que le hacía llegar a través de un mensajero.

Su abuela Augusta lo tuvo siempre en el mayor desprecio; no solía hablarle más que en contadas ocasiones, y todos sus avisos se los hacía llegar por medio de notas duras y lacónicas (Suet., Cl. III).

En cuanto a sus hermanos, Germánico y Livila, Claudio tuvo la «desgracia» de tener un hermano que contaba con todas las cualidades del ideal romano que él no poseía y que aumentaban todavía más su problema.

7 Es la pérdida de la facultad de realizar movimientos voluntarios para un fin determinado o de la comprensión del uso de objetos ordinarios.

${ }^{8}$ Es el movimiento anormal debido a una excesiva y/o inapropiada actividad muscular, que altera o llega a interrumpir la realización de los movimientos voluntarios.

${ }^{9}$ Los niños con alguna deficiencia, «diferentes", que necesitan más cuidados son muy susceptibles a ser maltratados.

10 Druso habría fallecido a consecuencia de una caída de caballo en septiembre del año 9 a.C.

11 Su abuela materna, Octavia, había fallecido un año antes de su nacimiento. 
Violencia y maltrato en la familia romana y sus consecuencias...

Es de sobra conocido que Germánico tuvo la suerte de poseer todas las virtudes físicas y espirituales, y en una medida mayor a la que nadie gozó jamás: una belleza y una fortaleza extraordinarias, un ingenio que sobresalía en la elocuencia y la erudición tanto en griego como en latín, una bondad excepcional, y una admirable y eficaz inclinación a granjearse la amistad de los hombres y a conquistar su afecto. (Suet., Cl. III).

La bondad que acompañó a Germánico no fue el caso de Livila que cuando oyó que un día su hermano sería emperador, abominó públicamente y en voz alta de la suerte tan miserable e indigna que le estaba reservada al pueblo romano (Suet., Cl. III 2).

Augusto, que sentía total aversión hacia los enanos y las personas que sufrían cualquier tipo de malformación física porque los consideraba seres de mal agüero (Suet., Aug. XCII 2), profesaba por el nieto de su hermana cierta simpatía y no le dejaba de sorprender su capacidad de razonamiento. En alguna ocasión, cuando Livia se encontraba ausente, invitaba a su sobrino-nieto a comer y fue en esos encuentros cuando tuvo la ocasión de conocerle mejor.

Durante tu ausencia —le escribe a su mujer-invitaré cada día a comer al joven Tiberio, para que no lo haga solo con sus queridos Sulpicio y Atenodoro. Me gustaría que eligiera con más cuidado y menos a la ligera un compañero del que pudiera imitar los gestos, el porte y la manera de caminar (Suet., Cl. IV 5). En otra carta, Augusto le confiesa a su esposa su sorpresa de que su «nieto Tiberio haya podido agradarme cuando declamaba, pues no veo cómo un hombre que se expresa con tan poca claridad puede decir claramente lo que se debe decir cuando declama» (Ibídem. 6).

Este trato más cercano con Augusto no supuso, sin embargo, una mejora en las condiciones de vida de Claudio pues, mientras él tenía como preceptor a aquel bárbaro, Augusto se preocupaba de atender las necesidades de los reyes aliados de Roma fijando habitualmente un tutor a los que aún eran niños o sufrían trastornos mentales e incluso educándolos junto con sus propios hijos. El único cargo que le permitió ejercer fue el de sacerdote augur y en su testamento se limitó a nombrarle heredero en tercer grado, como si fuese un extraño, y a concederle la sexta parte de su herencia, que no superaría los ochocientos mil sestercios (Suet., Cl. IV 7). Esto supuso una gran decepción para él y viendo que no podría nunca alcanzar un cargo público, decidió aislarse y buscar refugio en sus jardines, en la finca que poseía a las afueras de Roma o en la de Campania (Suet., Cl. V).

Claudio fue objeto de vejaciones y burlas no sólo por los miembros de su familia sino incluso también por el personal del palacio. Si llegaba tarde a cenar, nadie quería hacerle sitio y si lo hacían era a regañadientes y después de haberle hecho recorrer todo el triclinium. Cuando se dormía después de comer le molestaban arrojándole huesos de aceitunas o de dátiles, o le ponían chinelas en las manos para que se frotara la cara con ellas cuando se despertara. Hasta los bufones le convertían en objeto de sus «bromas» despertándole con un látigo o una palmeta (Suet., Cl. VIII).

La minusvalía física de Claudio hizo pensar a su familia que sus problemas, además de físicos, eran psíquicos también por lo que decidieron tenerlo lo más 
oculto posible. Porque, como afirmaba Augusto, «no hay que proporcionar a los hombres que tienen por costumbre burlarse de estas cosas y ridiculizarlas, la ocasión de reírse no sólo de él, sino también de nosotros» (Suet., Cl. IV 2).

De esta manera, Claudio no pudo ser protagonista en ceremonias tan significativas en la vida de un adolescente como la toma de la toga viril. En un acto en que los padres romanos organizaban toda una serie de celebraciones para festejar ese paso de la infancia a la vida adulta de los hijos, Claudio, el día en que tomó la toga viril, fue llevado en litera al Capitolio hacia la media noche, sin la ceremonia habitual (Suet., Cl. II 2). En otra ocasión, con motivo de unos juegos organizados en honor de su padre, se le permitió presidirlos pero oculto bajo un capuchón y habitualmente se le prohibía asistir a los juegos del circo desde el pulvinar.

La llegada a la adolescencia de Claudio supuso un problema añadido. Cuando vistió por primera vez la toga viril, nadie fue capaz de determinar si tenía sus capacidades mermadas o no, ni de decidir sobre su futuro viendo que, por sus características físicas, no podía seguir el cursus honorum habitual.

Cumpliendo tu encargo, mi querida Livia, hablé con tu hijo mayor acerca de lo que debía hacerse con tu nieto Tiberio durante los juegos de Marte. Los dos estamos de acuerdo, por otra parte, en que debemos decidir de una vez por todas qué criterio hemos de seguir respecto a él. Pues, si es apto, por decirlo así, en todos los sentidos, ¿qué motivo tenemos para dudar en promocionarlo haciéndole pasar gradualmente por las mismas etapas que hemos hecho pasar a su hermano? (Suet., $\mathrm{Cl}$. IV).

Sus biógrafos critican su desmesurada afición al juego y su cercanía a la gente de baja estofa (Suet., Cl. V 40). Apartado de los círculos sociales a los que pertenecía, Claudio debió de encontrar entre estas personas de condición social mucho más baja que la suya la compañía o al menos un ambiente menos hostil que el que tenía junto con su familia. Estos casos de manifiesto rechazo crean una tendencia al aislamiento porque no se debe olvidar que muchos pacientes del síndrome de Little no tienen mermada su capacidad intelectual y son plenamente conscientes de su realidad.

Los dados fueron una de sus grandes pasiones. Hasta el punto que, no sólo llegó a publicar un libro, sino que cuando viajaba procuraba colocar el tablero de forma tal que pudiese continuar jugando durante el viaje (Suet., $\mathrm{Cl}$. XXXIII 2). Sobre este tema cabrían dos observaciones: Primero, la influencia del entorno familiar en la conducta de los miembros más jóvenes. Esto es lo que comenta Suetonio de Augusto:

En cuanto a su fama de jugador, no la temió en absoluto, y jugó sin disimulo y a las claras, para divertirse, incluso en su vejez, (...) En una carta de su puño y letra dice: «Comí, mi querido Tiberio, con los mismos; (...) Durante la comida estuvimos jugando como viejos tanto ayer como hoy; tirábamos los dados ${ }^{12}(. .$.$) (Suet., Aug. LXXI 1).$

Dice en otra carta: «Nosotros, mi querido Tiberio, hemos pasado bastante agradablemente las Quincatrias, jugando, en efecto, durante todos estos días (...)» (Suet., Aug. LXXI 3).

\footnotetext{
12 Se refiere a las tabas, tali.
} 

pues.

El juego era una actividad habitual en la familia a la que Claudio no era ajeno,

Un segundo factor condicionante a la afición de Claudio por el juego debió de ser su imposibilidad de realizar cualquier actividad que requiriera esfuerzo físico como jugar a la pelota (expulsim ludere, trigon, harpastum,...), al escondite, la gallinita ciega, el basileus o la olla ${ }^{13}$. Su falta de psicomotricidad le obligaría a tener que entretenerse con juegos más sedentarios como las tabas (tali), los dados o el tres en raya.

Claudio, como hemos visto, no sufría ningún tipo de discapacidad intelectual. Incapaz de poder hacer actividades que requiriesen esfuerzo físico ni de tener los mismos entretenimientos que cualquier chico de su edad, se dedicó al estudió del griego y etrusco (de esta lengua es la última persona que se sabe que la conociese) y siendo todavía adolescente comenzó a escribir una historia de Roma siguiendo los consejos de Tito Livio y Suspicio Flavo. Pero a pesar de ello no logró mayor consideración ni mejora en sus expectativas de futuro.

Sin embargo, desde niño se aplicó con entusiasmo a los estudios liberales, y a menudo publicó incluso sus ensayos sobre todas estas materias (Suet, $\mathrm{Cl}$., 2).

Su manera convulsa de comer es, probablemente, consecuencia de una lesión en el hipotálamo causada por la misma isquemia que provocó el Síndrome de Little. El Hipotálamo, a pesar de su pequeño tamaño (apenas pesa $4 \mathrm{gr}$.), está considerado como un importante controlador de diversas funciones vegetativas entre ellas la regulación del metabolismo de los hidratos de carbono, de las grasas y proteínas, del sueño y ciertas funciones psíquicas y motoras como el lagrimeo o la salivación. La disfunción del hipotálamo provocaría en Claudio, entre otras cosas, la sensación constante de tener el estómago vacío ${ }^{14}$. Según Suetonio, rara vez se retiraba sin tener el estómago tan lleno que tenían que introducirle una pluma hasta la garganta para descargárselo (Suet., Cl. XXXIII). Prueba de su condición de comedor compulsivo era su incapacidad para dejar de comer a pesar de sufrir tal dolor de estómago que en más de una ocasión le hizo plantearse el suicidio (Suet., Cl. XXXI).

Siempre con ganas de comer y beber en cualquier circunstancia y lugar, un día que se hallaba juzgando en el Foro de Augusto y le llegó el olor de la comida que estaban preparando para los Salios en el cercano templo de Marte, abandonó el tribunal, subió hasta donde estaban los sacerdotes, y se sentó a la mesa con ellos. (Suet., Cl. XXXIII 2).

\footnotetext{
${ }^{13}$ En el juego de «el basileus» la distracción consistía en pegar al jugador más débil, conocido como «sarnoso», mientras en el de la «Olla» un pobre participante recibía los golpes de los demás hasta que conseguía atrapar a otro para que ocupase su lugar.

14 Las fuentes médicas consultadas apuntarían al hipotálamo como probable causa de los trastornos de Claudio, que justificaría, además de su falta de sensación de saciedad, los desórdenes del sueño. Sin embargo, no se descartan otros diagnósticos como una hiperfagia causada por un hipertiroidismo. Agradecemos al Dr. Ignacio Catalá Serra, médico del servicio de gástrico del hospital Arnau de Vilanova, su diagnóstico y orientaciones sobre este punto.
} 
En dos detalles la descripción de Suetonio no parece coincidir con los síntomas de un paciente de parálisis cerebral: mientras que este tipo de enfermos suele tener un crecimiento menor que el resto de la población, Suetonio afirmaba que su figura no carecía de prestancia ni de nobleza, pero eso cuando estaba de pie o sentado, y especialmente cuando descansaba, pues era un hombre alto y corpulento, de bello aspecto y hermosos cabellos blancos (Suet., CI. XXIX).

Tampoco en la parcela sexual parece haber coincidencia. Mientras los estudios actuales reflejan un mejor deseo sexual en este tipo de pacientes, Suetonio sostiene que sentía una extraordinaria pasión por las mujeres, y no tuvo ninguna relación homosexual.

La consecuencia de todo el maltrato que sufrió durante su infancia y adolescencia hicieron de él, ya como adulto, una persona, según siempre la descripción de Suetonio, variable, asustadiza, desconfiada y lo mismo frívola que comedida $^{15}$.

Fuera cónsul o no, administró justicia con extrema diligencia (...) No siempre se atuvo a lo prescrito por las leyes, sino que atenuó la dureza o la indulgencia de muchas de ellas conforme al bien y a la justicia, según sus impresiones (...) (Suet., Cl. XIV).

Cuidó siempre de Roma y de su abastecimiento con la mayor solicitud. (...) Con ocasión de una gran escasez de víveres provocada por una serie de malas cosechas (...) ideó todo tipo de soluciones para hacer llegar víveres incluso durante el invierno (Suet., $\mathrm{Cl}$. XVIII).

Sin embargo...

En los procesos que él instruía y sentenciaba, se mostraba increíblemente variable; unas veces era prudente y avisado, otras irreflexivo e impetuoso, y en ocasiones de una frivolidad rayana en la locura. (Suet., Cl. XV).

Pero era, ante todo, medroso de desconfiado. (...), no se atrevía a ir a los banquetes si no era rodeado por sus guardias (...) no visitaba tampoco a ningún enfermo sin haber hecho explorar antes su dormitorio y tentar y sacudir los colchones y la ropa de cama. (Suet., Cl. XXXV 1).

${ }^{15}$ El problema de personalidad puede estar relacionado, en palabras de la profesora M. ${ }^{\text {a José }}$ Díaz-Aguado Jalón a la que agradecemos sus orientaciones a este respecto, con dificultades en los modelos y expectativas básicos que tienen su primer origen en las relaciones de apego con las personas encargadas de proteger al niño. Existe una relación entre el estilo de apego desarrollado durante la infancia y ciertos comportamientos durante la edad adulta. El apego evitativo es el que se da cuando el cuidador deja de atender constantemente las señales de necesidad de protección del niño, y eso no le permite el desarrollo del sentimiento de confianza que necesita. Sería el caso de Claudio. Se sienten inseguros hacia los demás y esperan ser desplazados sobre la base de las experiencias pasadas de abandono (MIKULINCER: 1998). Según John Bowlby, pionero en examinar el rol que juegan los estilos de apego en la experiencia de rabia o enojo, las personas con estilo de apego evitativo tienen más propensión al enojo, caracterizándose por metas destructivas, frecuentes episodios de ira y otras emociones negativas. Sobre el maltrato infantil y sus consecuencias: FRANCHI, C., CALAM, R.:1988; VERDUGO ALONSO, M.: 1998. 
Aunque Suetonio, que no fue contemporáneo suyo ${ }^{16}$, lo describe en ocasiones como cruel, en Claudio no se dio esa situación tan habitual en la que un niño maltratado se convierte en un padre maltratador. Los testimonios de Suetonio nos lo presentan como un padre preocupado por la educación de sus hijos, que gustaba de comer junto a ellos y en ningún caso aparecen en él signos de violencia o desprecio.

En todas sus cenas admitía también a sus hijos, junto niños y niñas de noble linaje que comían según la costumbre sentados a los pies de los lechos. (Suet., $\mathrm{Cl}$.

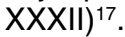

Sin embargo, no evitó que su hija pequeña, Octavia ${ }^{18}$, fuese una niña que a los trece años era incapaz de demostrar sentimiento alguno a causa de todo lo que había presenciado ${ }^{19}$.

A pesar de su joven edad, había aprendido a disimular el dolor, el amor y todos los afectos (Tac., Ann. XIII 16.4).

Octavia había sido prometida en matrimonio a Nerón siendo aún una niña y, según Tácito, el día de sus bodas fue como su funeral, llevada a una casa en la que no vería más que duelos, arrebatado su padre con veneno y poco después su hermano (Tac., Ann. XIV 63).

Claudio no fue, pues, el único miembro de la familia imperial en sufrir malos tratos o en no recibir la atención que un niño requiere. En las conductas desviadas que presentan, por ejemplo, Cayo Calígula o Lucio Domicio (Nerón) tienen mucho que ver, además de sus problemas neurológico/psiquiátricos, el abandono afectivo, el abuso, el mal ejemplo visto en los adultos o las situaciones traumáticas vividas durante la infancia.

En Capri, [Calígula ${ }^{20}$ ] a pesar de todas las asechanzas que le tendieron para incitarle y forzarle a prorrumpir en quejas, no dio jamás pretexto alguno, como si se le hubiera borrado por completo de la memoria la desgracia de los suyos y a ninguno de ellos le hubiera ocurrido nada; pasaba incluso por alto, con un disimulo increíble, lo que él mismo tenía que aguantar (...) (Suet., Gai. X 2).

Este es un breve resumen de la descripción que de la infancia del emperador Tiberio hace Suetonio:

Sus primeros años y su niñez fueron penosos y agitados, pues siempre acompañaba a sus padres en su huída (...) Llevado también a través de Sicilia y de Acaya

${ }^{16}$ Cayo Suetonio nació alrededor del año 70 d.C., es decir, unos quince años después de la muerte de Claudio en el 54 d.C.

17 Era habitual que los niños comieran junto a los adultos en una mesa aparte. El menú también era diferente, más apropiado a su edad. (Tac., Ann. XIII 16.2; Suet., Aug. LXIV 3).

18 Hija de Claudio y Mesalina, contrajo matrimonio con Nerón en el 53 d.C.

${ }^{19}$ Los testigos de violencia familiar tienen más posibilidades que otros en volverse ansiosos, reservados y pueden acabar considerando la violencia, incluso, como la forma apropiada de resolver los conflictos. KIMMEL et al.: 1998.

${ }^{20}$ Cuando tenía diecinueve años, Gayo pasó a vivir con su tío-abuelo, Tiberio, en Capri. 
(...) al partir de allí de noche corrió peligro de perder la vida, pues de repente estalló por todas partes un incendio en los bosques (...) A los nueve años perdió a su pa$\mathrm{dre}^{21}$, cuyo elogio fúnebre pronunció en la tribuna de las arengas (Suet., Tib. VI 1-6).

Después del divorcio de sus padres, Tiberio y su hermano Druso fueron criados y educados en casa de su padre. Cuando éste falleció, los dos niños se fueron a vivir con su madre, Livia Drusila, y su padrastro, Octavio.

\section{CONCLUSIÓN}

La psicología dice que las experiencias tempranas quedan y dejan huella aunque de manera inconsciente, y configuran la manera de ser. El maltrato constante y la discriminación por su minusvalía física a los que fue sometido Tiberio Claudio durante su infancia, adolescencia y gran parte de su vida adulta hicieron de él, por un lado, una persona variable, asustadiza e insegura y, por otro, un ser que intentó su superación personal, de innegables inquietudes culturales, y diligente y eficaz a la hora de gobernar.

Los efectos psicológicos que los malos tratos tienen sobre los adolescentes son diversos sin darse un único patrón pero, en cualquier caso, las consecuencias casi siempre son negativas ${ }^{22}$. Los adolescentes romanos, y concretamente los miembros de la familia Julio-Claudia, reaccionaron de formas muy diversas ante la realidad que les tocó vivir: desde el silencio de Octavia al fingimiento de Claudio o la memoria selectiva de Calígula. Esto debería de ser un motivo a tener en cuenta a la hora de intentar comprender y juzgar el comportamiento de ciertos personajes históricos.

\section{BIBLIOGRAFÍA}

ALCATUD MARÍN, F. (director) (2007): La parálisis infantil. Unidad de Investigación ACCESO. Departamento de Psicología evolutiva y de la Educación. Facultad de Psicología. Universidad de Valencia. http://acceso.uv.es/pci/PCl.html

BJERKLIE, D. (2006): «How the teen brain Works», Time Magazine, September 8. http://time.blogs.com/daily_rx/2006/09/attack_of_the_t.html.

BOWLBY, J. (1969): Attachment and Loss, Vol. 1: Attachment. Londres, Hogarth Press and the Institute of Psycho-Analysis.

DAL BIANCO, P. (1947): «Kaiser Claudius, einer patholographischen Analyse», Wien KIinische Wschr 59, pp. 597-601.

DENUELLE, P. (1974) : Maldie de Little, étude étiologique, diagnostique et thérapeutique d'après 42 observations, (Tesis doctoral), Université de Lyon 1.

FRANCHI, C., CALAM, R. (1988): Child Abuse and its consequences: observatorial aproacheses. Cambridge, Cambridge University Press.

GOGTAY et alii. (2004): «Dynamic mappic of human cortical development during childhood through early adulthood", PNAS (Proceeding of the National Academy of Sciences of the United States of America) 21 (vol. 101), pp. 8174-8179.

21 Tiberio Claudio Nerón (85 a.C.-33 a.C.).

22 WICKS-NELSON, R y ISRAEL, A.C., op. cit. pp. 356-362. 
GREZE, J. (1971) : Contribution à l'étude de la maladie de Little.

LEON, E. F. (1948): «The imbecillitas of Emperor Claudius», Transactions and proceedings of the American Philological Association 79, pp. 79-86.

LITTLE, WJ. (1862): «On the influence of abnormal parturition, difficult labours, premature birth, and asphyxia neonatorum, on the mental and physical condition of the child, especially in relation to deformities", Trans Obstet Soc Lon 3, pp. 293-344.

MARTIN, R.F. (1989): «Les paradoxes de l'empereur Claude», Revue des Etudes Latines 67, pp. 149-162.

- (1998): Los doce Césares. Del mito a la realidad. Madrid, Alberabán.

MIKULINCER, M. (1998): «Adult attachment style and individual differences in functional versus dysfunctional experiences of anger», Journal of Personality and Social Psychology 74 (2), pp. 513-524.

SCHWIENBACHER, I; FENDT, M.; RICHARDSON, R.; SCHNITZLER, H. (2004): «Temporary inactivation of the nucleus accumbens disrupts acquisition and expression of fearpotentiated startle in rats», Brain Res 1027 (1-2), pp. 87-93.

SUETONIO TRANQUILO, Cayo, (1992): Vida de los doce Césares, (Introducción de Antonio Ramírez de Verger, traducción y notas de Rosa M. ${ }^{a}$ Agudo Cubas). Madrid: Biblioteca Clásica Gredos n. ${ }^{\circ} 167-168$.

TÁCITO, Cornelio, (1991): Anales (Introducción, traducción y notas de José Luís Moralejo). Madrid: Editorial Gredos n. ${ }^{\circ} 19$.

VERDUGO ALONSO, M.A. (1998): El dolor invisible de la infancia: una lectura ecosistemática del maltrato infantil. Barcelona, Paidós Ibérica.

WALLIS, C. (2004): «What makes teens tick», Time 163, n. ${ }^{\circ}$ 19, pp. 56-65.

WICKS-NELSON, R \& ISRAEL, A.C. (1997): Psicología del niño y del adolescente. Madrid, Pearson Education. 